\title{
Utilization of curcumine and nanocurcumine compounds in
} cancer therapy

\author{
Vajiheh Alinezhad ${ }^{1}$, Heshmatollah Alinezhad ${ }^{1}$, Ramin Ataee ${ }^{2 *}$, Amin Ataie ${ }^{3}$ \\ ${ }^{1}$ Faculty of Chemistry, University of Mazandaran, Babolsar, Iran \\ ${ }^{2}$ Thallassemia Research Center, Pharmaceutical Sciences Research Center, Hemoglobiopathy Institute, Mazandaran University of Medical \\ Sciences, Sari, Iran \\ ${ }^{3}$ Deparment of Pharmacology and Physiology, Babol University of Medical Sciences, Babol, Iran
}

\begin{tabular}{|c|c|}
\hline & A B S T RACT \\
\hline $\begin{array}{l}\text { *Corresponding author: } \\
\text { raminataee1349@gmail.com }\end{array}$ & \multirow{5}{*}{$\begin{array}{l}\text { Curcumin is a polyphenol extracted from the Curcuma plant. Curcumin has been used widely in } \\
\text { ayurvedic medicine for centuries; it has a variety of biological properties including anti-oxidant, } \\
\text { analgesic, anti-inflammatory and antiseptic activity. Curcumin has shown anti-cancer activities } \\
\text { through variety of biological pathways engaged in mutagenesis, oncogene expression, cell cycle } \\
\text { regulation, apoptosis, tumorigenesis and metastasis. Curcumin has proved anti-proliferative effect in } \\
\text { many cancers, and is an inhibitor of the transcription factor NF-kB and downstream gene products } \\
\text { (including c-myc, Bcl-2, COX-2, NOS, Cyclin D1, TNF- } \alpha \text {, interleukins and MMP-9). Furthermore, } \\
\text { curcumin affects a variety of growth factor receptors and cell adhesion molecules involved in tumor } \\
\text { growth, angiogenesis and metastasis. However, a limiting factor is its extremely low bioavailability } \\
\text { which hinders its use as therapeutic agent. Therefore, many technologies have been developed to } \\
\text { overcome this limitation. We summarize to develop curcumin delivery aims and increase solubility for } \\
\text { improving curcumin bioavailability and anticancer potential for therapy. }\end{array}$} \\
\hline Article history: & \\
\hline & \\
\hline Accepted: Sep 28, 2017 & \\
\hline $\begin{array}{l}\text { Keywords: } \\
\text { Curcumin, apoptosis, } \\
\text { anti-proliferative, } \\
\text { metastasis, anti- } \\
\text { inflammatory, antisept }\end{array}$ & \\
\hline
\end{tabular}

Citation: Pharm Biomed Res 2017;3(3):1-11.

\section{Introduction}

The continual reduction in cancer death rates over two decades has resulted in an overall drop of $23 \%$, resulting in more than 1.7 million cancer deaths prevented. In spite of this development, cancer is now the leading cause of death for much of the US population (1,2). In 2016, an estimated 1,685,210 new cases of cancer will be diagnosed in the United States and 595,690 people will die from the illness.

Current chemotherapeutic factors, such as alkylating agents, anti-metabolites, topoimerase inhibitors, and DNA intercalates, target a particular route, so finally dwindles tumor size but often fails to eradicate tumors or prevent their recurrence. Repeated therapy with these agents eventually causes tumors that become resistant to the chemotherapies. So, it is important to identify 'natural products 'which have growth inhibitory and apoptosis induction properties on human cancer cells and that target multiple cellular signaling paths without resulting in toxicity issues in normal cells. A comprehensive review of the literature texts characterized curcumin as an excellent molecule among many naturally occurring compounds for cancer therapy (3). There are three primary curcuminoids which constitute curcumin:

curcumin (curcumin A, 75\%), demethoxycurcumin (curcumin B, 20\%), and bisdemethoxycurcumin (curcumin C, 5\%; and our own analysis) (Fig. 1) (4).<smiles>COc1cc(/C=C/C(=O)CC(=O)/C=C/c2ccc(O)c(OC)c2)ccc1O</smiles><smiles>COc1cc(/C=C/C(=O)CC(=O)/C=C/c2ccc(O)cc2)ccc1O</smiles><smiles>O=C(/C=C/c1ccc(O)cc1)CC(=O)/C=C/c1ccc(O)cc1</smiles>

Figure 1 Chemical structures of curcumin (A), demethoxycurcumin (B) and bisdemethoxycurcumin (C).

Chemically, curcumin is a diarylheptanoid, belongs to the group of curcuminoids, that are natural phenols responsible for turmeric's' yellow color. This is a tautomeric combination existing in enolic form in organic solvents and as a keto form in water (5). 


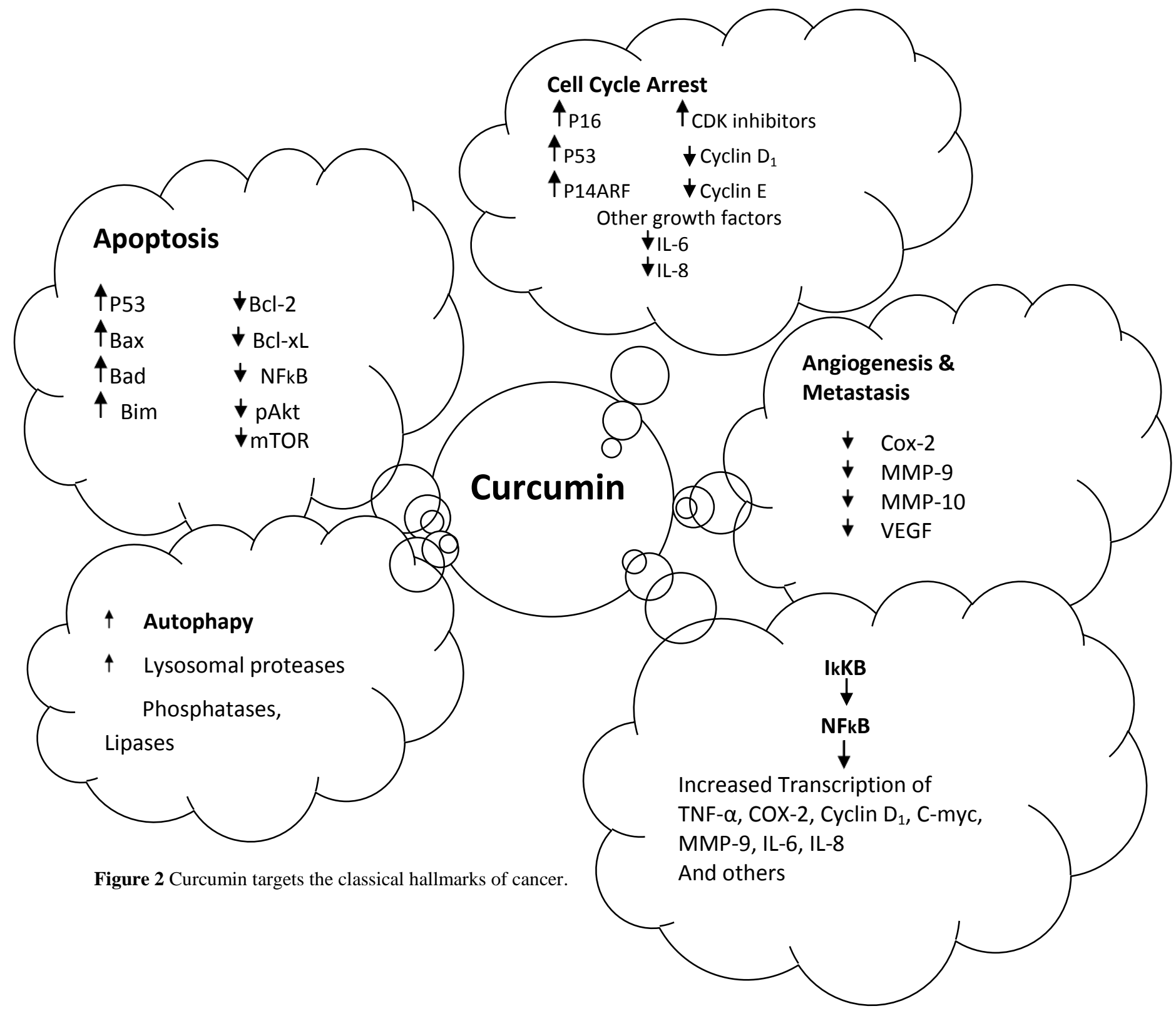

Research during the last two decades has indicated curcumin to be a strong antioxidant, antiinflammatory, anti-proliferative, anti-metastatic, antiangiogenic, anti-diabetic, hepatoprotective, antiatherosclerotic, anti-thrombotic, and anti-arthritic agent in cell culture and animal studies (6). A serious limiting factor of curcumin is its low solubility in water (i.e. $0.0004 \mathrm{mg} / \mathrm{ml}$ at $\mathrm{pH} 7.3$ ) and soluble curcumin molecules are much sensitive at physiological $\mathrm{pH}$ (7-8). Removing the limiting factors of curcumin to improve its bioavailability, should prevent from degradation and metabolism, and increase its targeting capacity toward cancer tumor(s). Curcumin is available in various types of nanoparticle (NPs), such as polymer NPs, polymeric micelles, liposome phospholipid, nanomicroemulsions, nanogels, solid lipid NPs, polymer conjugates, self-assemblies, and so on, which are proper for the delivery of an active form of curcumin to tumors (9).

\section{Anti-cancer Activity}

Curcumin has been examined in multiple human carcinomas including melanoma, head and neck, 
breast, colon, pancreatic, prostate and ovarian cancers (10-18). It is good point of curcumin that can inhibit all primary abilities of cancer cells and restrict the consequence of tumor progression in the host (19). The expression of nuclear factor NFKB that controls cell proliferation, metastasis, angiogenesis, apoptosis and resistance to chemotherapy, is prevented by curcumin (20). Curcumin-induced down-regulation of NF- $\kappa \mathrm{B}$ is mediated via suppression of I $\kappa \mathrm{B}$ kinase activation. The proliferation signaling cascades such as PI3K, AKT, mTOR, AP1 (JUN and FOS), JNK, JAK-STAT, PKC, CMYC, MAPK, ELK, CDKs, iNOS and Wnt/ $\beta$ catenin which are also suppressed by curcumin further confirmed that it is one of the crucial molecule that hinders cancer promotion by targeting multiple cell proliferation signaling. Curcumin also down-regulates the expression of Cyclin D1, the proto-oncogenes that are overexpressed in various types of cancer and plays a definite role in cell cycle progression and proliferation $(21,22)$. TP53 is the most important protein that manages on central regulatory circuits which controls the decision of cells whether to proliferate or undergo active ageing and provokes apoptosis schedule. Different researches in vitro and in vivo have been proved that curcumin upregulates the expression of TP53 and provokes apoptosis (23). On the other hand, curcumin also inhibits phosphorylation of RB (Retinoblastoma), another serious tumor suppressor protein that also plays an important role in cell cycle process (24). Curcumin inhibits EGF- and EGFR-mediated signaling path that is overexpressed in breast tumor and is involved in cancer advancement (25, 26).

Curcumin also blocks excessive TGF $\beta$ receptor signaling that prompts epithelial to mesenchymal transition during offensive and metastasis $(27,28)$. During tumor development, TP53 is reduced so hindering its critical action as damage sensor and activator of apoptosis-inducing circuitry. On the other hand, tumors may attain similar sequels by increasing expression of anti-apoptotic regulators (BCL-2, BCL-XL) or survival signals (IGF1/2), or down regulating pro-apoptotic agents (BAX, BIM, PUMA), or by short-circuiting the extrinsic ligandinduced death path (29). Curcumin prompts both TP53-dependent and -independent cancer cell apoptosis. curcumin upregulates the pro-apoptotic molecules such as BAX, BIM, PUMA, whereas, curcumin down-regulates anti-apoptotic partners like BCL2, BCL-XL, Survivin,so that, curcumin simultaneously activates Caspases and induces apoptosis or programmed cell death $(30,31)$. Lysosomal proteases, phosphatases and lipases that provoke autophagy-mediated cell death, are activated by curcumin $(32,33)$. Generally, neo- angiogenesis process is managed by the angiogenic factors such as VEGF and angiopoietin inducement. VEGF and angiopoietin overexpression factors are restricted by curcumin, that hinders angiogenesis process by clipping food and oxygen supply to the cancer cells (34). VEGF receptor (VEGFR1 and VEGFR2) expression is inhibited by curcumin, thereby blocking VEGF/VEGFR-mediated signalling pathway to restrict angiogenesis (23). Besides human telomerase (hTERT) activities, downregulation hTERT-mRNA expression leading to telomere shortening is inhibited by curcumin. Thus curcumin targets telomerase activities and controls replicative cell senescence and mortality which finally regulate uncontrolled cell proliferative potential of cancer (35). Curcumin down-regulates several attacks, cell adhesion and extracellular matrix molecules such as matrix metalloprotease, CCRX4, COX2, ELAM1, ECAM1 which are essential for maintaining metastasis (36). In addition, several research also proposed that curcumin hampers the activities of SLUG, SNAIL, FAK, TWIST and other urgent transcription factors that play a crucial role in metastasis process (37). Lately, it was indicated that curcumin inhibits breast cancer stem cell migration by amplifying E-cadherin/beta-catenin negative feedback ring (Fig. 2) (38).

\section{Curcumin nanoformulations}

In spite of, curcumin activities as a potent immunemodulator, there are some of the major hurdles that hinders its efficacy as a chemotherapeutic drug against cancer such as poor water solubility, low bioavailability, lack of dose-response proportionality, uncontrolled precipitation, use of excessive co-solvents, necessity of extreme condition to solubilize (basic or acidic) and incompatibility to the patients $(39,40)$. Nanotechnology-based drug delivery systems have proven to be reliable and promising approach, to overcome this inconveniences. Poor bioavailability, enhance biological activities and also selectively target cancer cells are improved by nanotechnology-based drug delivery systems. Now it is possible to deliver the active pharmaceutical component as reduced nano-sized particles, ranging in size from 10 to $1000 \mathrm{~nm}$, to enhance systematic bioavailability of higher molecular weight drugs. The nanotechnology-based drug delivery system has proven as a most effective manner to successfully deliver insoluble drugs with enhanced bioavailability (41). The decrease of particle size of active ingredients significantly enhances the dissolution rate resulting in higher bioavailability. Various kinds of nanoparticles are being expanded for successful encapsulation of curcumin. These contain liposomes, micelles, nanoparticles, nanoemulsions, nanogels, nanocrystal suspensions, 
phytosome complexes, inclusion complexes and dendrimer/dimers (42). Lately, pure curcumin nanoparticles have been developed that are 50 times more effective than normal curcumin, with increased bioavailability, instead of carrier-based nano formulations. These curcumin nanoparticles confine tumor-induced Treg cells by hindering several Treg markers and restore immune surveillance in tumor-bearing mice (43).

Albeit, nanotechnology based drug delivery system has been confirmed as a major effective and promising approach towards successful cancer therapy but there are certain limitations. Some problems like possibility of drug targeting, drugloading capacity, in vivo fate of the carrier-molecule conjugates (interactions with the biological microenvironment, rate of disintegration and accumulation in organs), toxic effects of the carrier molecule or its metabolites, its large scale manufacture, stability during long-term storage and overall production costs are difficult to deal with. Particularly, a crucial parameter in the body is the toxic effects of the nano-formulations. Albeit, the carrier materials are examined for toxicity and biocompatibility, nevertheless the qualities of the nano particles often differ from bulk material. Therefore, it is necessary that rigorous and specialized tests take for determining the toxicities of the carrier molecules, its metabolites and surfactants, before approval for use (39).

\section{Curcumin emulsions, liposome and phospholipid formulation}

Eucalyptol-based curcumin microemulsions have extremely high permeability and flux with average solubility of curcumin compared with lots of oleic acid- and esteem oil-based microemulsions (40). The enhanced penetration capacity of this applied curcumin microemulsion formulation have been observed also its effect on the cellular structure of skin. Moreover, a new microemulsion (approximately $10 \mathrm{~nm}$ ) combination of limonene, polysorbate 80, ethanol and water promotes curcumin presence in skin (9.29-29.99 $\mu \mathrm{g}$ in an area of $0.785 \mathrm{~cm}^{2}$ ) (41). Coacervation technique in the presence of polymeric non-ionic surfactants prepared Myristic, palmitic, stearic and behenic acids (i.e., fatty acids; FA)-based solid lipid NPs (42). This manner at first weigh down to $28-81 \%$ with a $500-\mathrm{nm}$ particle size. After hydrolysis, this diameter size diminished to $300 \mathrm{~nm}$. A stable selfmicroemulsifying drug delivery system (SMEDDS) containing $20 \%$ ethanol, $60 \%$ Cremophor RH $40 \AA$ and $20 \%$ isopropyl myristate was able to encapsulate $50 \mathrm{mg} / \mathrm{ml}$ of curcumin and to discharge it totally in $10 \mathrm{~min}$ (43-45).

Curcumin microemulsions made ready in the presence of ethyl oleate and isopropyl myristate result in homogeneous, yellow, transparent solutions, whiles soybean oil and peppermint oil are turbid in nature (45). The emulsion crosslinking method suitable for lung targeting formulated extremely flat surface gelatin microspheres with $75.5 \%$ curcumin encapsulation efficiency (45). These spheres are 5-30 $\mu \mathrm{m}$ in diameter, with $50 \%$ curcumin discharge within $22 \mathrm{~h}$ for urgent remedial effects. Co-administration of curcumin and paclitaxel nanoemulsion formulations are capable of conquering multidrug resistance in SKOV3 (TR) human ovarian adenocarcinoma cells by hampering NF-KB activity, downregulating P-gp and promoting apoptotic answer (46). Moreover, the bioavailability of paclitaxel up to 5.2 -fold is increased by curcumin nano emulsion and there is a 3.2-fold increase in its accumulation at the tumor site in an oral administration to SKOV3 tumorbearing xenograft mice models, that resulted from downregulation of intestinal P-gp and cytochrome P450 3A2 (CYP3A2) protein levels [47]. Liposomes constitute artificial phospholipid vesicles, considered to be biologically safe, biocompatible and preserve drugs from external stimuli. It has been known that a prolonged absorption capacity of curcumin can be obtained by dissolving, mixing or complexing it with several types of phospholipid. Sou et al. (48) have successfully made ready a curcumin lipid formulation using 1,2-dimyristoylsn-glycero-3-phosphocholine (DMPC) and an anionic amphiphilic, L-glutamic acid, N-(3-carboxy1-oxopropyl)-1,5-dihexadecyl ester (SA).

Intravenous administration of this formulation in rats indicated no acute response in circulating blood cells and extremely of the curcumin accumulated in bone marrow and spleen tissues. A recent pharmacokinetic research of solid lipid curcumin NPs in patients with osteosarcoma introduced up to $31.42-41.15 \mathrm{ng} / \mathrm{ml}$ of curcumin within $4 \mathrm{~h}$ of $2000-4000 \mathrm{mg}$ oral dose treatment (49). More importantly, the patients did not experience any side effects. In another research, solid lipid NPs with $134 \mathrm{~nm}$ and 84\% encapsulation of curcumin displayed slow discharge of curcumin over a week (50). High oral bioavailability in plasma of male wistar rats is resulted in by this formulation. Curcumin solid lipid NPs at every single oral dose of $1,12.5,25$ and $50 \mathrm{mg} / \mathrm{kg}$ pharmacokinetics in rats attained a maximum concentration $\left(\mathrm{C}_{\max }\right)$ of bioavailability in serum $1.00 \pm 0.01,7.87 \pm 3.02,8.00 \pm 1.87$, and $14.29 \pm 0.15$ $\mu \mathrm{g} / \mathrm{ml}$, respectively, whiles free curcumin was only present at $0.292 \pm 0.06 \mu \mathrm{g} / \mathrm{ml}$, even at a dose of 50 $\mathrm{mg} / \mathrm{kg}$. This enhancement in bioavailability result in improvement of curcumin absorption.

A comparative survey showed the uptake of liposome- and serum albumin-loaded curcumin formations in normal spleen lymphocytes and EL4 
lymphoma cells via fluid phase pinocytosis and membrane fusion, respectively (51). Curcuminloaded liposomal formulation was a more useful delivery transporter and further noteworthy absorption and fluorescence levels in lymphoma cells compared with normal cells. Li et al. (52) assessed the lipid curcumin ratio (10:1 wt./wt.) on different pancreatic carcinoma cell lines, including ASPC-1, BxPC-3, Capan-1, Capan-2, HS766-T and MiapaCa2, and showed an inhibition concentration of IC50 at 2.0-37.8 $\mu \mathrm{M}$, whiles IC90 was 6.75-94.5 $\mu \mathrm{M}$, as determined by cytotoxicity. Recently, curcumin was successfully inserted in eggphosphatidylcholine (EPC) liposomes at a 1:14 molar ratio (curcumin:liposome). A twofold improvement in concentration of curcumin was present in rat plasma with the lecithin curcumin formulation compared with a curcumin and curcumin-lecithin mixture after recommending of a single oral dose of $100 \mathrm{mg}$ curcumin $/ \mathrm{kg}$ body weight [53]. Narayanan et al. (54) provided an interesting vision of the use of curcumin and resveratrol in liposome to consider their combined effects on: (i) cell progress, apoptosis and the cell cycle; and (ii) on activated p-Akt, cyclin D1, mammalian target of rapamycin (m-TOR) and androgen receptor (AR) proteins involved in tumor progression of PTEM-CaP8 prostate cancer cells. Totally, this combined formulation remarkably reduced incidence of prostatic adenocarcinoma in vivo . Transferrin-solid lipid NPs maintained $83 \%$ of curcumin for 6 months and particularly, targeted MCF-7 breast cancer cells four- and five fold in 24 and $48 \mathrm{~h}$ therapy, respectively (55). Transferrinmediated endocytosis was verified, which promotes a kind of reactive oxygen (ROS) and timedependant apoptosis by transferrin-solid lipid curcumin NPs. Curcumin structural aspects facilitated nanodisk NPs containing a disk-shaped lipid bilayer formation generally stabilized by an apolipoprotein scaffold (56). The effectiveness of anticancer properties of this lipid curcumin formulation in human hepatocellular carcinoma (HepG2) was significant over that of free curcumin.

\section{polymeric NPs.}

Poly (lactic-co-glycolic acid) (PLGA) is an extensive choice in the production of a variety of biomedical devices due to the its biodegradability and biocompatibility. Several different types of PLGA NP for curcumin encapsulation have been explored because of producing a safe carrier. Curcuminencapsulated PLGA NPs has been prepared to use a simple solid-oil-water solvent evaporation method (57). with the surfactant concentration and sonication time control the particle size. Afterward, solvent evaporation method is designed to regulate the curcumin-encapsulated PLGA NPs via a lower particle size, increased intracellular uptake and antibody conjugation aspects (58). Grabovac and Bernkop studied first the development of surfacemodified PLGA NPs with a thiolated chitosan [59]. Level functionalization of curcumin-loaded PLGA NPs by a bis (sulfosuccinimidyl) suberate (BS3) has facilitated conjugation of annexin A2 and resulted in an effective target treatment of curcumin to annexin A2-positive MDA-MB-231 cancer cells [60]. Shahani and Panyam (61) planned a sustained and injectable microparticle formulation with higher curcumin loading capacity (i.e. $38.1 \mathrm{mg} / 100 \mathrm{mg}$ of particles; $\quad 76.2 \%$ encapsulation efficiency) contrasted with many formulations. A developed glutathione-s-transferase (GST) activity in liver was distinguished with injectable microparticles and this observed phenomenon was steady over 4 weeks. Usually a potent endogenous defense mechanism against carcinogens is demonstrated by GST activity. In vivo researches in mice which applied this formulation, has resulted a tenfold increase in blood, brain and lungs curcumin, compared with which dissolved in PEG 400 formulation (62). Curcumin-loaded PLGA has been designed by Tsai et al. (63) which increased the half-life of curcumin in the cerebral cortex and hippocampus significantly from 2.32 to 19.9 and 7.56 to $16.7 \mathrm{~min}$, respectively. Moreover, retention time values of the cerebral cortex and the hippocampus were increased almost 2.0- and 1.8fold, respectively. With this nano-formulation, the curcumin plasma levels were slightly higher. Nano formulations based on dextran sulfate-chitosan are considered to be biocompatible materials which can be applied for oral, intravenous and controlled delivery aims. Other research quantified the cellular uptake of curcumin encapsulated in dextran sulfatechitosan NPs with applying a spectrophotometric method in L929, MCF-7, PC3 and MG 63 cells (64). Moreover, a cytotoxicity evaluation and fluorescence-activated cell sorting (FACS) investigation proposed that anticancer activity of this formulation is high in MCF-7 compared with other cancer cells. A curcumin analog encapsulated in polycaprolactone (PCL) NPs (approximately 650 $\mathrm{nm}$ ) controlled intravenously to male Wistar rats demonstrated higher intracellular surface in liver cells, which proposed that this formulation can be used to target the liver (65). Multi-drug resistance cancer cells (K562 cells) are treated more effectively by co-encapsulation of curcumin and doxorubicin in polymer NPs (66). Primary curcumin release from NPs downregulated MDR1 and BCL-2 expression and hindered the nuclear efflux mechanism; the subsequent release of doxorubicin provoked cancer cell death. A patented technology described by synthesis of curcuminencapsulated chitosan NPs demonstrated to be safe 
in mice and rat researches at a dose of $4 \mathrm{mg}$ and 40 $\mathrm{mg}$ of formulations, respectively, for 14 days (67).

\section{Micelles}

A micelle, regularly is a surfactant molecule aggregate dispersed in a liquid colloid. It is a nanosized vesicular membrane which became it soluble in water by gathering the hydrophilic heads outer surface in contact with the solvent and hydrophobic tails inside and is known as emulsifications. Micelles are lipid molecules which organize themselves in a spherical form in aqueous solutions with a very narrow range from 10 to $100 \mathrm{~nm}$ in size, that makes them more stable through dilution in biological fluids (68). The structure or morphology of micelles is from amphiphilic block copolymers both spherical, rodlike, and tarlike, as well as vesicles. The selfassembly of amphiphilic block copolymer is a reversible method, and the shape differs with the copolymers' composition and length ratio (69). The performance of micelles is based on amphiphilic block copolymers, that come together to form a nanosized core/shell structure in aqueous media. The hydrophilic shell surface stabilizes the hydrophobic core and result in the polymers water soluble while the hydrophobic core area distributes like a pool for hydrophobic drugs. Water-insoluble drugs such as curcumin can serve by polymeric micelles as transmitters, which can raise the drug's efficiency by targeting certain cells or organs so that, fewer drugs gather in healthy tissues and their toxicity decreases and occasionally higher doses can be administered (70). With this attention, Liu et al. (71) prepared curcumin-loaded biodegradable self-assembled polymeric micelles by solid dispersion method was simple and easy to scale up and has overcome the poor water solubility of curcumin. Release profile, between quick release of free curcumin and much slower and sustained release of curcumin-loaded micelles indicated a significant difference. Moreover, Raveendran et al. (72) investigated that preparation of curcuminloaded micelles based on amphiphilic Pluronic/polycaprolactone block copolymer, has been confirmed to be efficient in increasing curcumin's aqueous solubility. Other researches also evaluated on highly surface-active compounds such as poloxamer or Pluronic which can selfassemble into spherical micelle. In vitro results indicated that spherical curcumin-loaded mixed micelles might serve as potential nanocarriers to amend the solubility and biological activity of curcumin (73-74). In another research, encapsulation within the micelles increased the aqueous solubility of curcumin (75). Solubilization was directly related to the compatibility between the solubilizate and polycaprolactone as determined by the Flory-Huggins interaction parameter. Curcumin tended to interact with polycaprolactone serving as a core embraced by polyethylene glycol as a shell was proposed by molecular modeling research. Moreover, the structure of modified $\varepsilon$-polylysine micelles and their utilization in improving cellular antioxidant activity of curcuminoids was shown by $\mathrm{Yu}$ et al. (76). Results of these studies showed that curcuminoids can improve their water solubility and cellular antioxidative activity compared with free curcuminoids when encapsulated via modified $\varepsilon$-polylysine micelles. It was suggested that for delivering poorly soluble drugs such as curcumin may use these new biopolymer micelles. To overcome the poor water solubility of curcumin, another synthesized curcumin in sodium dodecyl sulfate and cetyltrimethylammonium bromide has indicated antioxidative effects of curcumin analogues against the free-radical-induced peroxidation of linoleic acid in these micelles (77, 78). Due to their higher solubility than free curcumin, kinetic analysis of the antioxidation processes proved that these compounds showed incredibly higher antioxidative activity (77). Several issues indicated micelle stability, the rateof copolymer biodegradation, and drug diffusion control release from micelles.

By the way,the potential of the two most common Pluronic triblock copolymer micelles, Pluronic F127 and F68, for curcumin encapsulation efficiency and stability was reported by Sahu et al. (79). Pluronic F127 indicated better encapsulation efficiency and good stability for long-term storage than Pluronic F68. Atomic force microscopy (AFM) research demonstrated that the drug-encapsulated micelles are spherical in shape with diameters below $100 \mathrm{~nm}$. Pluronic-encapsulated curcumin showed slower and sustained release of curcumin from the micelles and substantial anticancer activity in comparison with free curcumin during in vitro cytotoxicity study. Moreover, a natural protein core-based polymeric micelle were reported by Podaralla et al. (80) which applied for delivering of hydrophobic anticancer drugs, specifically curcumin. Also it was produced new biodegradable micelles by conjugating methoxy polyethylene glycol and zein, a biodegradable hydrophobic plant protein which then encapsulating with curcumin. Polyethylene glycol zein micelles maintained the curcumin release up to $24 \mathrm{~h}$ in vitro and remarkably enhanced its aqueous solubility and stability with 3fold reduction in IC50 of curcumin. So, whereas the curcumin is finely protected from possible inactivation by their micellar environment, its retention and bioavailability can also be extended. Because of modifying the 
pharmacokinetics of curcumin, poly(D,L-lactide-coglycolide)-b-poly(ethylene glycol)-b-poly(D,Llactide-co-glycolide) (PLGA-PEG-PLGA) with micelles were synthesized by Song et al (81). PLGAPEG-PLGA micelles provided higher area under the concentration curve (AUC) and increased residence time, clearance, and distribution half-life in comparison with curcumin solution. The extension of half-life, enhanced residence time, and decreased total clearance showed which curcumin-loaded micelles could prolong acting time of curcumin in vivo. These results may be in relation with curcumin location within the micelles and enhanced viscosity of copolymer solution at body temperature. The change of AUC proved that the curcumin-loaded micelles provided higher bioavailability than curcumin solution, and the biodistribution research indicated that the micelles had diminished drug uptake by liver and spleen and intensified drug distribution in lung and brain. These outcomes proposed that PLGA-PEG-PLGA micelles would be a potential bearer for curcumin. Moreover,the pharmacokinetics of both solubilized curcumin and its polymeric micellar formulation in rats via using a simple, rapid, and reliable HPLC method were proved by Ma et al. (73). They concluded that curcumin's half-life and distribution volume increased by encapsulation of curcumin in the polymeric micellar formulation. Moreover, physicochemical characteristics, concentration, and location within the micelles can be affected by curcumin-micelles. The polymeric micelles have a prolonged circulation time because of the small size and hydrophilic layer which reduces the drug uptake via the mononuclear phagocyte system (82). Encapsulated curcumin in cationic micelles suppresses alkaline hydrolysis that was investigated in three types of micelles composed of the cationic surfactants cetyltrimethylammonium bromide (CTAB) and dodecyltrimethylammonium bromide (DTAB) and the anionic surfactant sodium dodecyl sulfate (SDS) which were reported by Leung et al. (83).

Curcumin underwent rapid decomposition in the SDS micellar solution with alkaline hydrolysis at $\mathrm{pH}$ of 13 , whiles it was significantly suppressed with performance of suppression close to $90 \%$ in the presence of either CTAB or DTAB micelles. The results of the fluorescence spectroscopic studies showed that curcumin is separated from the SDS micelles to the aqueous phase at this $\mathrm{pH}$ while curcumin stays on encapsulated in CTAB and DTAB micelles at $\mathrm{pH}$ 13. The lack of encapsulation and stability in the SDS micellar solution led rapid hydrolysis of curcumin. Other curcumin-loaded micelles properties were indicated by some other studies. The sensitive fluorometric method for the determination of curcumin using the increase of mixed micelle was introduced by Wang et al. (84). This method had the benefits of high sensitivity, selectivity, and stability. Mixed micelle of sodium dodecylbenzenesulfonate and cetyltrimethylammonium bromide (SDBS-CTAB) greatly increased the fluorescence of curcumin. This research showed that fluorescence quantum yield of curcumin in SDBS-CTAB micelle was about 55fold bigger than that of aqueous solution containing $1.0 \%$ ethanol, that was in agreement with their fluorescence intensity ratio. As a result, curcumin could be used as a fluorophore in fluorescence polarization anisotropy measurement in order to critical micellar concentration of surfactant and to check their interaction. Moreover, femtosecond fluorescence upconversion experiments on the naturally occurring medicinal pigment, curcumin, in anionic, cationic, and neutral micelles were performed by Adhikary et al. (85). These micelles were formed of SDS, dodecyltrimethylammonium bromide (DTAB), and Triton X-100. They showed the curcumin's excited-state kinetics in micelles with fast (3-8 ps) and slow (50-80 ps) components. While deuteration of curcumin had a negligible effect on the fast component, the slow component displayed a pronounced isotope impact of approximately 1.6, it shows that micelle-captured curcumin undergoes excited-state intramolecular hydrogen atom transfer. In addition, curcumin to phosphatidylcholine micelles followed by fluorescence measurements had been attached by Began et al. (86). Curcumin in aqueous solution did not prevent dioxygenation of fatty acids by lipoxygenase 1 , but it prevented the oxidation of fatty acids when bound to phosphatidylcholine micelles. Results showed that for $50 \%$ inhibition of linoleic acid peroxidation requires $8.6 \mu \mathrm{M}$ of curcumin bound to the phosphatidylcholine micelles. Line Weaver-Burk plan analysis had demonstrated that curcumin is a competitive inhibitor of lipoxygenase 1 with $\mathrm{Ki}$ of $1.7 \mu \mathrm{M}$ for linoleic acid and $4.3 \mu \mathrm{M}$ for arachidonic acid, respectively. Using spectroscopic measurement, they showed that the inhibition of lipoxygenase 1 activity by curcumin could be because of binding to active center iron and curcumin after binding to the phosphatidylcholine micelles acts as an inhibitor of lipoxygenase 1. In a recent research,with fluorescent probe determined the critical micelle concentration of the amphiphilic polymer. Results demonstrated that Pluronic/polycaprolactone micelles may be an acceptable candidate for curcumin delivery to cancer cells of colorectal adenocarcinoma (72).

In another pharmacokinetic study, higher concentration and longer retention time in plasma 
and tumor sites were indicated by curcumin micelles, so they had stronger inhibitory effects on proliferation, migration, invasion, and tube formation of carcinoma cells than free curcumin; for instance, curcumin micelles were shown to be more effective, probably because of higher concentration in preventing tumor growth and long-term survival in both subcutaneous and pulmonary metastatic tumor models (87). Check the impact of micelles on cytotoxicity of curcumin, specifically in cancer therapy, in vitro research by Raveendran et al. (72) indicated that Pluronic/polycaprolactone micelles could be an acceptable candidate for curcumin delivery to cancer cells due to the cytotoxicity and cellular uptake of the curcumin-loaded micelles in colorectal adenocarcinoma cells. The study by Wang et al. [88] showed that the encapsulated curcumin keeps its potent antitumor effects; Nevertheless, curcumin-loaded micelles were more efficient in inhibiting tumor growth and spontaneous pulmonary metastasis in subcutaneous 4T1 breast tumor model and longterm survival of tumor-bearing mice. Tumors of curcumin-loaded micelle-treated mice had more apoptotic cells, fewer microvessels, and fewer proliferation-positive cells also were showed by Immunofluorescent and immunohistochemical research (88). Moreover, methoxypolyethylene glycol-polylactic acid (mPEG-PLA) micelle to several curcumin molecules had been conjugated by Yang et al. (89); the cytotoxicity research outcomes demonstrated that the effect of IC50 of mPEG-PLA-Tris-curcumin on human hepatocellular carcinoma cells was similar to unmodified curcumin. The cellular uptake research showed that these bearers can successfully transport the drug to the cytoplasm of hepatic cells. Micelles containing several drug molecules were an effective means to increase loading and intracellular transfer of lowpotency curcumin (89). Moreover,the curcumin encapsulated in methoxy poly(ethylene glycol)/poly-epsilon-caprolactone diblock copolymeric (MePEG/PCL) micelle, with change the copolymer ratio (40:60 MePEG/PCL ratio was selected due to its high encapsulation), had expanded bioavailability because of strong uptake, 2.95 times more, with comparative cytotoxic effects by induction of apoptosis in contrary with unmodified curcumin at equimolar concentrations were reported by Mohanty et al. (90). Generally, these data certainly demonstrated the commitment of a micellar system for efficient solubilization, stabilization, and controlled delivery of the hydrophobic drug, such as curcumin, for cancer treatment. Briefly, curcumin-loaded micelles can increase the drug's efficiency by targeting definite cells and outcome in less drug aggregation in healthy tissues and decrease of toxicity. Curcumin's aqueous solubility and much slower and sustained release of drug created by curcumin-loaded micelles also obtain for use in several conditions. The survival and bioavailability of curcumin could be high since the curcumin is preserved from possible inactivation by its micellar surroundings. Locating the curcumin in the micelles can also increase half-life and residence time and reduce total clearance leading to lengthening of acting time of curcumin. Curcumin micelles could be impressed by physicochemical features containing their size and electrical charges, concentration, and location within the micelles.

\section{Conclusion}

Curcumin is found to be an extremely helpful anticancer factor, targeting different paths connected with cancer development. Researchers continue to disclose new ways of its action and its interaction that with the immune system is coming out as an important factor to its anti-cancer properties. The need for tumor cells to evade the immune system during successful tumor progression in the body is now considered a new characteristic of cancer. Several advanced studies in the past decade have gradually published curcumin as a powerful immune-modulator. Albeit, several reports have proposed a general immunosuppressive role of curcumin and its capability to diminish cell proliferation in immune cell in isolation; specific reports show that curcumin increases anti-tumor immunity via different mechanisms, as discussed in this review. So modulation of the immune system shows to be another important strategy by which curcumin neutralizes cancer growth. This furthermore claims its effect as an anti-cancer factor and points out the need to develop it as an adjuvant chemotherapeutic factor. This necessitates the improvement of nano-based strategies for suitable delivery and increased bioavailability of curcumin, that may eventually lead to its use as an appropriate chemotherapeutic factor.

\section{Conflict of interest}

The authors report no conflicts of interest.

\section{Financial disclosure}

No financial supports have been granted by any agency to conduct this publication.

\section{References}

1. Keyvani V, Kerachian M A. The effect of fasting on the important molecular mechanisms related to cancer treatment. J Fasting Health 2014;2:113-18

2. Walker B, Figgs L W, Zahm S H. Differences in cancer incidence, mortality, and survival between African Americans and whites. Environ Health Perspect1995; 103(Suppl 8):275-81. 
3. Manolova Y, Deneva V, Antonov L, Drakalska E, Momekova D, Lambov N. The effect of the water on the curcumin tautomerism: a quantitative approach. Spectrochim Acta A Mol Biomol Spectrosc 2014;132:815-20

4. Anand P, Thomas SG, Kunnumakkara AB, Sundaram C Harikumar KB, Sung B, et al. Biological activities of curcumin and its analogues (Congeners) made by man and Mother Nature. Biochem pharmacol 2008;76:1590-611.

5. Tønnesen H. Studies on curcumin and curcuminoids. XVI. Effect of curcumin analogs on hyaluronic acid degradation in vitro. Int J pharm 1989;51:259-61.

6. Tønnesen H. Solubility, chemical and photochemical stability of curcumin in surfactant solutions. Studies of curcumin and curcuminoids, XXVIII. Die Pharmazie 2002;57:820-24.

7. Muqbil I, Masood A, Sarkar FH, Mohammad RM, Azimi AH Progress in nanotechnology based approaches to enhance the potential of chemopreventive agents. Cancers 2011;3:428-45.

8. Aggarwal S, Takada Y, Singh S, Myers JN, Aggarwal BB. Inhibition of growth and survival of human head and neck squamous cell carcinoma cells by curcumin via modulation of nuclear factor- $\kappa \mathrm{B}$ signaling. International J Cancer 2004;111;67992.

9. LoTempio MM, Veena MS, Steele HL, Ramamurthy B Ramalingam TS, Cohen AN. Curcumin suppresses growth of head and neck squamous cell carcinoma. Clin Cancer Res 2005; 11:6994-7002

10. Wang D, Veena MS, Stevenson K, Tang C, HoB A, Suh JD, et al Liposome-encapsulated curcumin suppresses growth of head and neck squamous cell carcinoma in vitro and in xenografts through the inhibition of nuclear factor $\mathrm{\kappa B}$ by an AKT-independent pathway. Clin Cancer Res 2008;14:6228-36.

11. Mukhopadhyay A, Bueso RC, Chatterjee D, Pantazis P, Aggarwa BB. Curcumin downregulates cell survival mechanisms in human prostate cancer cell lines. Oncogene 2001;20:7597-609.

12. Mehta K, Pantazis P, McQueen T, Aggarwal BB. Antiproliferative effect of curcumin (diferuloylmethane) against human breast tumor cell lines. Anti-cancer drugs 1997; 8:470-81.

13. Hanif R, Qiao L, Shiff SJ, Rigas B. Curcumin, a natural plant phenolic food additive, inhibits cell proliferation and induces cell cycle changes in colon adenocarcinoma cell lines by a prostaglandin-independent pathway. J Lab Clin Med 1997; 130:576-84

14. Elattar TM, Virji AS. The inhibitory effect of curcumin, genistein, quercetin and cisplatin on the growth of oral cancer cells in vitro. Anticancer Res 2000;20:1733-8.

15. Lin YG, Kunnumakkara AB, Nair A, Merritt WM, Han LY, Armaiz-Pena GN, et al. Curcumin inhibits tumor growth and angiogenesis in ovarian carcinoma by targeting the nuclear factor$\kappa$ B pathway. Clin Cancer Res 2007;13:3423-30.

16. Siwak DR, ShishodiaS, Aggarwal BB, Kurzrock R. Curcumininduced antiproliferative and proapoptotic effects in melanoma cells are associated with suppression of IкB kinase and nuclear factor $\kappa \mathrm{B}$ activity and are independent of the B-Raf/mitogenactivated/extracellular signal-regulated protein kinase pathway and the Akt pathway. Cancer 2005;104:879-90.

17. Hanahan D, Weinberg RA. The hallmarks of cancer. Cell 2000;100:57-70.

18. Bharti AC, Donato N, Singh S, Aggarwal BB. Curcumin (diferuloylmethane) down-regulates the constitutive activation of nuclear factor $-\kappa \mathrm{B}$ and $\mathrm{I} \kappa \mathrm{B} \alpha$ kinase in human multiple myeloma cells, leading to suppression of proliferation and induction of apoptosis. Blood 2003;101;1053-62.

19. Choudhuri TS, Das T, Sa G. Curcumin selectively induces apoptosis in deregulated cyclin D1-expressed cells at G2 phase of cell cycle in a p53-dependent manner. J Biolog Chem 2005; 280; 20059-68.

20. Kunnumakkara AB, Aggarwal BB. Curcumin inhibits proliferation, invasion, angiogenesis and metastasis of different cancers through interaction with multiple cell signaling proteins. Cancer lett $2008 ; 269: 199-25$.

21. Tsyetkoy P, Asher G, Reiss V, Shaul Y, Sachs L, Lotem J. Inhibition of $\mathrm{NAD}(\mathrm{P}) \mathrm{H}$ : quinone oxidoreductase 1 activity and induction of p53 degradation by the natural phenolic compound curcumin. Proc Natl Acad Sci USA 2005;102:5535-40.

22. Aggarwal BB, Banerjee S, Bharadwaj U, Sung B, Shishodia S, Shethi G. Curcumin induces degradation of cyclin E expression through ubiquitin-dependent pathway and up-regulates cyclindependent kinase inhibitors p21 and p27 in multiple human tumor cell lines. Biochem pharmacol 2007;73:1024-32.

23. Zhan Y, Chen Y, Liu R, Zhang H, Zhang Y. Potentiation of paclitaxel activity by curcumin in human breast cancer cell by modulating apoptosis and inhibiting EGFR signaling. Arch Pharmacal Res 2014;37:1086-95.

24. Sun XD, Liu X E, Huang DS. Curcumin induces apoptosis of triple-negative breast cancer cells by inhibition of EGFR expression. Molec Med Rep 2012;6:1267-70.

25. Gaedeke J, Noble N A, Border WA. Curcumin blocks multiple sites of the TGF- $\beta$ signaling cascade in renal cells. Kidney Int 2004;66;112-20.

26. Ataie A, Shadifar M, Ataee R. Polyphenolic Antioxidants and Neuronal Regeneration. Basic Clin Neurosci. 2016;7:81-90.

27. Chakraborty J, Banerjee S, Ray P, Hossain D MS, Bhattacharyya S, Adhikary A, et al. Gain of cellular adaptation due to prolonged p53 impairment leads to functional switchover from p53 to p73 during DNA damage in acute myeloid leukemia cells. J Biolog Chem 2010;285: 33104-112

28. Shankar S, Chen Q, Sarva K, Siddiqui I, Srivastava RK. Curcumin enhances the apoptosis-inducing potential of TRAIL in prostate cancer cells: molecular mechanisms of apoptosis, migration and angiogenesis. J Mol Signal 2007;2:10.

29. Pal S, Choudhuri T, Chattopadhyay S, Bhattacharya A, Datta G K, Das $\mathrm{T}$, et al. Mechanisms of curcumin-induced apoptosis of Ehrlich's ascites carcinoma cells. Biochem Biophys Res Commun 2001;288:658-65.

30. Karunagaran D, Rashmi A, Kumar TR. Induction of apoptosis by curcumin and its implications for cancer therapy. Current Cancer Drug Targets 2005;5:117-29.

31. Hasima N, Ozpolat B. Regulation of autophagy by polyphenolic compounds as a potential therapeutic strategy for cancer. Cell death Dis $2014 ; 5: \mathrm{e} 1509$.

32. Tan W, Lu J, Huang M, Li Y, Chem M, Wu G, et al. Anti-cancer natural products isolated from chinese medicinal herbs. Chin Med 2011;6:27.

33. Lee JH, Chung IK. Curcumin inhibits nuclear localization of telomerase by dissociating the Hsp90 co-chaperone p23 from hTERT. Cancer Lett 2010;290:76-86.

34. Shehzad A, Lee YS. Molecular mechanisms of curcumin action: signal transduction. Biofactors 2013;39:27-36

35. Lee AYL, Fan CC, Chen YA, Cheng CW,Sung YJ, Hsu CP, et al. Curcumin inhibits invasiveness and epithelial-mesenchymal transition in oral squamous cell carcinoma through reducing matrix metalloproteinase 2, 9 and modulating p53-E-cadherin pathway. Integr Cancer Ther 2015;14:484-90.

36. Mukherijee S, Mazumdar M, Chakraborty S, Manna A, Saha S, Khan $\mathrm{P}$, et al. Curcumin inhibits breast cancer stem cell migration by amplifying the E-cadherin/ $\beta$-catenin negative feedback loop. Stem Cell Res Ther 2014;5:116.

37. Anand P, Kunnumakkara AB, Newman RA, Aggarwal B. Bioavailability of curcumin: problems and promises. Mol Pharm 2007; 4:807-18

38. Bisht S, Feldmann G, Ravi R, Karikar C, Maitra A. Polymeric nanoparticle-encapsulated curcumin ("nanocurcumin"): a novel strategy for human cancer therapy. J Nanobiotech 2007;5:3.

39. Bonifácio BV, da Silva P B, dos Santos Ramos M A, Negri KMS, Bauab TM, Chorilli M. Nanotechnology-based drug delivery systems and herbal medicines: a review. Int J Nanomed 2014; 9:115 .

40. Bonifácio BV, da Silva PB, dos Santos Ramos MA, Negri K MS, BauabT, MChorilliM. Nanotechnology-based drug delivery systems and herbal medicines: a review. International journal of nanomedicine2014; 9:1

41. Hossain D, Panda AK. MEK inhibition prevents tumour-shed transforming growth factor- $\beta$-induced T-regulatory cell augmentation in tumour milieu. Immunology2015;144-: 561-73.

42. Liu $\mathrm{CH}$, Chang FY. Development and characterization of eucalyptol microemulsions for topic delivery of curcumin. Chem 
Pharmaceut Bull 2011;59:172-78.

43. Liu $\mathrm{CH}$, Chang FY. Terpene microemulsions for transderma curcumin delivery: effects of terpenes and cosurfactants. Colloids Surf B Biointerfaces 2011; 82: 63-70.

44. Anoop K, Sharma N. Comparative efficacy of piperine and curcumin in deltamethrin induced splenic apoptosis and altered immune functions. Pestic Biochem Physiol 2015;119:16-27

45. Chirio D, Gallarate M, Peira E, Battaglia L, Serpe L, Trotta M. Formulation of curcumin-loaded solid lipid nanoparticles produced by fatty acids coacervation technique. $\mathrm{J}$ microencapsul 2011;28:537-48.

46. Wu X, Xu J, Huang X, Wen C. Self-microemulsifying drug delivery system improves curcumin dissolution and bioavailability. Drug Dev Int Pharm 2011;37:15-23.

47. Lin CC, Lin HY, Chen HC, Yu MW. Stability and characterisation of phospholipid-based curcumin-encapsulated microemulsions. Food Chem 2009; 116:923-28.

48. Cao FL, Xi YW, Tang L, Yu AH, Zhai GX. Preparation and characterization of curcumin loaded gelatin microspheres for lung targeting. J Chin Med Mat 2009:32;423-26.

49. Ganta S, Amiji M. Coadministration of paclitaxel and curcumin in nanoemulsion formulations to overcome multidrug resistance in tumor cells. Mol Pharm 2009;6:928-39.

50. Ganta S, Devalaally H, Amiji M. Curcumin enhances ora bioavailability and anti-tumor therapeutic efficacy of paclitaxel upon administration in nanoemulsion formulation. J Pharm Sci 2010;99:4630-41.

51. Sou K, Inenaga S, Takeoka S, Tsuchida E. Loading of curcumin into macrophages using lipid-based nanoparticles. Int $\mathrm{J}$ Pharm 2008;352: 287-93.

52. Gota VS, Maru GB, Soni TG, Gandhi TR, Kochar N, Agarwa MG. Safety and pharmacokinetics of a solid lipid curcumin particle formulation in osteosarcoma patients and healthy volunteers. J Agric Food Chem 2010;58:2095-99.

53. Kakkar V, Singh S, Singla D, Kuar IP. Exploring solid lipid nanoparticles to enhance the oral bioavailability of curcumin. Mol Nutr Food Res 2011;55:495-503.

54. Kunwar A, Barik A, Pandey R, Priyadarsini KI. Transport of liposomal and albumin loaded curcumin to living cells: an absorption and fluorescence spectroscopic study. Biochim Biophys Acta 2006;1760:1513-20

55. Kurzrock R., Li L.. Liposome-encapsulated curcumin: in vitro and in vivo effects on proliferation, apoptosis,signaling, and angiogenesis. In ASCO Annual Meeting Proceedings2005;. 23, 16 suppl:4091)

56. Jing Li a, Yu Zhoua, Wei Zhangb, Cuiyu Baoa, Zhigang X, Relief of oxidative stress and cardiomyocyte apoptosis by using curcumin nanoparticles. Colloids Surf B Biointerfaces 2017;153:174-82.

57. Pandelidou M, Dimas K, Georgopoulos A. Preparation and characterization of lyophilised egg PC liposomes incorporating curcumin and evaluation of its activity against colorectal cancer cel lines. J Nanosci Nanotech 2011;11:1259-66.

58. Narayanan NK, Nargi D, Randolph C, Narayanan BA. Liposome encapsulation of curcumin and resveratrol in combination reduces prostate cancer incidence in PTEN knockout mice. Int J Cancer 2009;125:1-8.

59. Mulik RS, Mönkkönen J, Juvonen RO, MahadikKR, Paradkar AR Transferrin mediated solid lipid nanoparticles containing curcumin: enhanced in vitro anticancer activity by induction of apoptosis. Int J Pharm 2010;398:190-203.

60. Ghosh M, Singh AT, Xu W, Sulchek T, Gordon LI, Ryan RO. Curcumin nanodisks: formulation and characterization. Nanomed Nanotech Biol Med 2011:7:162-7.

61. Mukerjee A, Vishwanatha JK. Formulation, characterization and evaluation of curcumin-loaded PLGA nanospheres for cancer therapy. Anticancer Res 2009; 29:3867-75.

62. Yallapu MM, Gupta BK, Jaggi M. Fabrication of curcumin encapsulated PLGA nanoparticles for improved therapeutic effects in metastatic cancer cells. $\mathbf{J}$ colloid interface science2010; 351:19 29.

63. Millotti G, Samberger C, Fohlich E, Bernkop Schnurch A.Chitosan-graft-6-mercaptonicotinic acid: synthesis, characterization, and biocompatibility. Biomacromolecules
$2009 \cdot 10 \cdot 3023-7$

64. Thamake SI, Raunt S, Ranjan AP, Grycznski Z, Vishwanatha JK .Surface functionalization of PLGA nanoparticles by non-covalent insertion of a homo-bifunctional spacer for active targeting in cancer therapy. Nanotechnology 2011;22:035101.

65. Shahani K, Panyam J.Highly loaded, sustained-release microparticles of curcumin for chemoprevention. J Pharm Sci 2011;100:2599-609.

66. Shahani K, Swaminathan SK, Freeman D, Blum A, Ma L, Panyam J. Injectable sustained release microparticles of curcumin: a new concept for cancer chemoprevention. Cancer Res 2010;70:4443-52.

67. Tsai TM, Chein CF, Lin LC, Tsi TH. Curcumin and its nanoformulation: the kinetics of tissue distribution and blood-brain barrier penetration. Int J Pharm 2011;416:331-8.

68. Anitha A, Deepagan VG, Rani VD, Menon D, Nair SV, Jayakuma R. Preparation, characterization, in vitro drug release and biological studies of curcumin loaded dextran sulphate-chitosan nanoparticles. Carbohydrate Polymers 2011;84:1158-64.

69. Anuradha CA, Aukunuru J. Preparation, characterisation and in vivo evaluation of bis-demethoxy curcumin analogue (BDMCA) nanoparticles. Tropic J of Pharm Res 2010;9:51-8

70. Misra R, Sahoo SK. Coformulation of doxorubicin and curcumin in poly (D, L-lactide-co-glycolide) nanoparticles suppresses the development of multidrug resistance in K562 cells. Mol Pharm 2011;8:852-66.

71. Shehzad A, Ul-Islam M, Wahid F, Lee YS. Multifunctiona polymeric nanocurcumin for cancer therapy. J Nanosci Nanotech 2014;14:803-14

72. Agil F, Munagala R, Jeyabalan J, Vadhanam F. Bioavailability of phytochemicals and its enhancement by drug delivery systems.Cancer letters 2013;334:133-41.

73. Cho HK, Cheong IW, Lee JM, Kim JH. Polymeric nanoparticles, micelles and polymersomes from amphiphilic block copolymer. Kor J Chem Engin 2010;27:731-40.

74. Jones MC, Leroux JC. Polymeric micelles-a new generation of colloidal drug carriers. Eur J Pharm Biopharm 1999;48:101-11.

75. Liu L, Sun L, Wu Q, Guo W, Li L, Chen Y, et al. Curcumin loaded polymeric micelles inhibit breast tumor growth and spontaneous pulmonary metastasis. Int J Pharm 2013;443:175-82

76. Raveendran R, Behuvaneshwar GS, Sharma CP In vitro cytotoxicity and cellular uptake of curcumin-loaded Pluronic/Polycaprolactone micelles in colorectal adenocarcinoma cells. J Biomater Applic 2013;27:811-27.

77. Yu H, Li J, Shi K, Huang Q. Structure of modified ع-polylysine micelles and their application in improving cellular antioxidant activity of curcuminoids. Food Funct 2011;2:373-80.

78. Dai F, Chen WF, Zhou B, Yang L, Liu ZL. Antioxidative Effects of Curcumin and its Analogues against the Free-radical-induced Peroxidation of Linoleic Acid in Micelles. Phytother Res 2009;23:1220-8

79. Mondal S, Ghosh S. Role of curcumin on the determination of the critical micellar concentration by absorbance, fluorescence and fluorescence anisotropy techniques. J Photochem Photobiol B 2012;115:9-15

80. Sahu A, Kasoju N, Goswami P Boara U Encapsulation of curcumin in Pluronic block copolymer micelles for drug delivery applications. J Biomat Applica 2011;25;619-39.

81. Podaralla S, Averineni R, Alqahtani M, Perumal O. Synthesis of novel biodegradable methoxy poly (ethylene glycol)-zein micelles for effective delivery of curcumin. Mol Pharm 2012;9:2778-86.

82. Song Z, Feng R, Sun M, Guo C, Gao Y, Li L, et al. Curcuminloaded PLGA-PEG-PLGA triblock copolymeric micelles: Preparation, pharmacokinetics and distribution in vivo. J Colloid Interface Sci 201;354:116-23

83. Ma Z, Haddadi A, Molavi O, Lavasanifar A, Lai R, Samuel J. Micelles of poly (ethylene oxide)-b-poly ( $\varepsilon$-caprolactone) as vehicles for the solubilization, stabilization, and controlled delivery of curcumin. J Biomed Mater Res 2008:86:300-10.

84. Yokoyama M. Clinical applications of polymeric micelle carrier systems in chemotherapy and image diagnosis of solid tumors. J Exp Clin Med 2011;3:151-8

85. Leung MH, Colangelo H, Kee TW. Encapsulation of curcumin in cationic micelles suppresses alkaline hydrolysis. Langmuir 
2008;24:5672-5.

86. Wang F, Wu X, Wang F, Liu S, Jia Z, Yang J. The sensitive fluorimetric method for the determination of curcumin using the enhancement of mixed micelle. J Fluoresc 2006;16:53-9.

87. Adhikary R, Carlson PJ, Kee TW, Petrich JW. Excited-state intramolecular hydrogen atom transfer of curcumin in surfactant micelles. J Physic Chem B 2010;114:2997-3004.

88. Began G, Sudharshan E, Appu Rao AG. Inhibition of lipoxygenase 1 by phosphatidylcholine micelles-bound urcumin. Lipids 1998;33:1223-8.

89. Wang F, Huang W, Jiang L, Tang B. Quantitative determination of proteins based on strong fluorescence enhancement in curcuminchitosan-proteins system. J Fluores 2012;22:615-22.

90. Yang R, Zhang S, Kong D, Gao , Zhao Y,Wang Z. Biodegradable polymer-curcumin conjugate micelles enhance the loading and delivery of low-potency curcumin. Pharm Res 2012;29:3512-25. 